\title{
DESIGN E INTERNET DAS COISAS EM PRODUTOS DE LINHA BRANCA NO BRASIL.
}

Carolina Haidee Bail Afonso Rosenmann (carolinahaidee@gmail.com) - Programa de Pós-Graduação em Design, Universidade Federal do Paraná.

Julia Letícia Sciamana (juliascimana@gmail.com) - Programa de Pós-Graduação em Design, Universidade Federal do Paraná

Valkiria Pedri Fialkowski (val.pedri@gmail.com) - Programa de Pós-Graduação em Design, Universidade Federal do Paraná

Virginia Souza de Carvalho Borges Kistmann (vkistmann@ufpr.br) - Programa de Pós-Graduação em Design, Universidade Federal do Paraná

\section{RESUMO}

Este artigo apresenta um estudo de campo sobre o uso da IoT em eletrodomésticos no Brasil. Esse setor vem incorporando a tecnologia denominada Internet of Things por oferecer novas funções que impactam o design. Ainda pouco explorada, ela vem se tornando um desafio para empresas. Um estudo exploratório não encontrou resultados bibliográficos e empíricos relativos à sua aplicação no mercado nacional. Portanto, este artigo tem como objetivo principal identificar as tendências de uso da IoT em produtos de linha branca no Brasil. Parte de uma revisão da literatura, seguida de uma pesquisa empírica. Foram pesquisados fabricantes de grande porte, sendo considerados: incidência, modo de uso da IoT, uso combinado e design. Os resultados apontam uma baixa incidência do uso da IoT dentre as empresas investigadas. Maior incidência dela foi encontrada junto a produtos pertencentes ao portfólio de maior valor agregado. Quanto ao modo de uso destacam-se aplicativos relacionados às funções de controle, programação e tempo. Quanto à relação entre aparelhos, verifica-se a tecnologia Bluetooth predominante nos achados, como intermediação entre os smartphones. Por fim, o resultado do estudo mostra que as empresas de linha branca exploram a incorporação dessa tecnologia, sem, no entanto, realmente isso ficar evidente no design.

Palavras-chave: Gestão de Design, Produtos, Linha Branca, Internet das Coisas.

Área: Gestão do Processo de Desenvolvimento de Produto.

\section{INTRODUÇÃO}

O avanço tecnológico tem oportunizado e popularizado o acesso à internet. Segundo o IBGE estima-se que, no Brasil, mais de $80,6 \%$ da população com mais de 10 anos utilizam microcomputadores para acesso à internet, 73,6 \% utilizam telefones móveis celular e 16,4\% tablets (IBGE, 2014). A conexão por meio destes dispositivos móveis tende a crescer cada vez mais e se estender para outros objetos. Para Santos et al (2017) a Internet das Coisas (Internet of Things - IoT) é uma extensão da Internet atual aos objetos quaisquer do cotidiano, mas com capacidade computacional e de comunicação, sendo que essa conexão viabiliza o controle remoto destes objetos.

Segundo Battaiola et.al (2014) alguns exemplos da IoT são eletrodomésticos como refrigeradores, fogões e máquinas de lavar roupas que estão sendo lançados pelas indústrias 
com conexão à internet e algumas novas funcionalidades relacionadas a essa conexão. Dessa maneira, estes produtos passam a estar conectados ao virtual para acesso remoto a fim de propiciar ao usuário maior conveniência, conforto ou economia.

A IoT pode mudar as relações sociais interligando real e virtual (GASPARETTO et al, 2016), dessa maneira surgem alguns desafios aos profissionais de design. Alguns desses desafios podem ser caracterizados pelos seguintes questionamentos: quais as técnicas e tecnologias utilizadas para a conexão destes produtos à internet, como viabilizar novas funcionalidades e serviços possibilitados por esta conexão, e como integrar os usuários a estas novas funcionalidades, considerando a comunicação destas e a experiência de interação dos usuários junto aos produtos.

A partir destes novos desafios e oportunidades que a IoT oferece, o profissional de design tem a necessidade de compreender as tendências para melhor proveito das possibilidades desta tecnologia emergente no Brasil. Porém, no mercado nacional, os estudos relacionados ao uso da IoT em linha branca com este intuito ainda são pouco explorados. Assim, apresenta-se como pergunta de pesquisa: quais as tendências no uso da IoT em produtos de linha branca no Brasil?

Portanto, este estudo apresenta inicialmente uma revisão da literatura sobre o termo Internet das Coisas (Internet of Things - IoT), assim como do contexto de aplicação deste conceito em novos produtos, objetivando embasar um estudo de campo a respeito do uso da IoT em produtos da linha branca no Brasil. A seguir, apresenta dados levantados em um estudo de campo, em que foram analisados de modo comparativo produtos de diferentes empresas produtoras de linha branca, com o objetivo de identificar tendências futuras da utilização da IoT nessa linha de produtos.

\section{IOT CONCEITOS E DESIGN}

Visando apresentar conceitos para embasar o estudo aqui proposto, este capítulo apresenta uma fundamentação teórica pautada em autores que vêm abordando em seus estudos a compreensão da IoT e sua relação com o design.

Segundo Ashton (2009 apud. SANTOS et al, 2017), o uso do termo Internet of Things (IoT) ocorreu pela primeira vez em seu trabalho intitulado de "I made at Procter \& Gamble" em 1999, sendo este mais relacionado ao uso da tecnologia. Contudo, somente a partir do ano de 2005 a expressão começou a tornar-se popular tanto para academia quanto para a indústria. Nesta época, segundo Santos et al (2017), as pesquisas buscavam assuntos referentes aos sensores sem fio, refletindo, assim, os avanços da automação residencial e industrial. Nos anos seguintes, o termo passou a ganhar notoriedade rapidamente, batendo recorde de buscas em 2010 (SANTOS et al, 2017).

O termo Internet das coisas, traduzido da sigla em inglês IoT (Internet of Things), é uma expressão coletiva usada para descrever a mudança de um modelo de interação tecnológica, no qual as pessoas passaram a interagir com objetos que possuem capacidade de comunicação entre si a partir de sensores incorporados e compartilhados em longas distâncias usando a internet (DESBARATS, 2017).

Sob o aspecto do modo de uso, segundo Lee et al (2017), a IoT pode ser vista como um cruzamento de diversas tecnologias, as quais se complementam para a viabilização da integração dos objetos entre os ambientes físico e virtual. Para sua prática, faz-se necessário ter no seu processo uma colaboração entre os vários campos do conhecimento como a telecomunicação, informática, eletrônica e a ciência social (LEE et al, 2017). Na IoT, segundo 
a autora, a unidade básica de hardware apresenta ao menos: a) uma unidade de processamento; b) uma unidade de memória; c) uma unidade de comunicação e; d) uma unidade de sensor(es) ou atuador(es). A transmissão das informações coletadas nessas unidades básicas entres os objetos denominados de Smart Objects (Objetos Inteligentes) pode ocorrer por meio do compartilhamento por Wi-Fi, Bluetooth, QR Codes, dentre outros (CANTANHEDE, SILVA, 2014).

Com respeito à relação entre os diferentes dispositivos, Santos et al. (2017) afirma que são diversas as técnicas usadas para conectar objetos inteligentes. Algumas das tecnologias usadas podem interligar aparelhos como smartphones, tablets e microcomputadores com outros aparelhos que possuam possibilidade de conexão para estes, que podem ser eletroeletrônicos em geral como os produtos os produtos de linha branca. A relação entre os aparelhos permite ampliar as potencialidades dos profissionais de design ao permitir o acesso a informações dos usuários, de forma a aumentar o conhecimento a respeito do uso e preferencias do produto (BATTAIOLA et al., 2014).

Quanto ao design, entende-se que o desenvolvimento de produtos e/ou serviços, que propõem o uso deste conceito de conectividade, deve estar, primeiramente, pautado no usuário. Dessa forma, para o campo do design isto significa desenvolver projetos interativos, em uma relação direta com as experiências do seu público-alvo, maximizando assim a aceitação desses novos produtos e/ou serviços no mercado (GASPARETTO et al, 2016). Em termos de comunicação, vale ressaltar que funciona como uma ponte entre a interface dos dispositivos interativos que irão se comunicar entre si por meio dessa rede com o usuário, permitindo assim que a comunicação entre os aparelhos propicie, também, uma experiência ao consumidor (GASPARETTO et al, 2016).

Segundo Gasparetto et al (2016), é essa cultura da experiência que determina a entrada das tecnologias no cotidiano das pessoas. No caso da IoT, essa atua como um fator exponencial no aumento das possibilidades de conexão, trazendo novos desafios para esta área. Devido a esses desafios há a necessidade da atuação do designer ao longo de todo o processo de desenvolvimento de um projeto de produto e/ou serviço interativo é ressaltada. A partir do design centrado no usuário, atividade permeada pela subjetividade do ser humano, busca-se propiciar experiências eficazes e positivas por parte dos consumidores.

Em suma, a IoT, ao conectar objetos com diferentes recursos a uma rede, potencializa o surgimento de novas aplicações. Segundo Santos et al (2017), os objetos ao promoverem comunicação entre usuários com os dispositivos, possibilitam não somente um aumento na facilidade e qualidade de vida destes, como também, permitem a coleta de dados e monitoramento dos mesmos. Desta forma, podemos entender que o seu uso tem influenciado o cotidiano tanto do indivíduo quanto dos negócios (LEE, 2017).

\section{MÉTODO}

A pesquisa que originou este artigo caracteriza-se como uma pesquisa exploratória, a qual buscou identificar as tendências do uso da IoT em produtos de linha branca no Brasil. Para isso, adotou um estudo de campo, que, embora semelhante a um levantamento, proporciona uma maior profundidade e flexibilidade na pesquisa, podendo até encaminhar-se na reformulação dos objetivos ao longo da mesma (GIL, 2002).

Neste artigo, o estudo de campo divide-se em duas etapas, sendo a primeira de cunho virtual, utilizando uma pesquisa documental na internet, e a segunda realizada de forma presencial, por meio de visitas à pontos de vendas físicos de eletrodomésticos na cidade de Curitiba, Paraná, realizado no ano de 2017 (FIALKOWSKI, 2017). Vale ressaltar que neste estudo, em 
nenhuma das etapas, foram analisados produtos que não apresentassem qualquer relação com o escopo de busca.

Para a etapa virtual, foram pesquisados os sites e portfólios de todas as marcas que comercializam atualmente produtos de linha branca no Brasil, procurando, portanto, evidenciar nos documentos encontrados as principais funcionalidades dos produtos que fazem uso da tecnologia de IoT. Ademais, complementou-se esta etapa com uma busca secundária em sites que continham informações relevantes acerca do funcionamento e uso desses eletrodomésticos. Como resultado da pesquisa virtual, pode-se então obter uma visão mais ampla e aprofundada das marcas e respectivos produtos que atualmente fazem uso da tecnologia da IoT incorporada ao seu funcionamento.

Por fim, com a finalidade de constatar e verificar as informações adquiridas dos produtos de linha branca que fazem uso da tecnologia de IoT na etapa virtual, foi realizado uma pesquisa presencial em quatro pontos de vendas de eletrodomésticos na cidade de Curitiba. Por meio desta pesquisa, pode-se confrontar a informação obtida pelos sites com os produtos físicos, e também analisar o uso da IoT nos produtos da linha branca comercializados no país.

No estudo presencial então, foram analisados produtos do mercado brasileiro de linha branca, por meio de observação do campo, que tivessem ocorrência do uso de algum sistema de IoT como evidenciado na literatura estudada. Ele foi realizado junto a revendedores de produtos da linha branca e considerou aqueles ofertados por empresas de grande porte, que foram: Electrolux, Brastemp, Midea, LG e Samsung. Vale ressaltar que, por meio do estudo realizado, não foram encontrados produtos com o uso do conceito de IoT para outras marcas de linha branca existentes no mercado brasileiro aqui apresentado. As visitas foram efetuadas em 4 revendedores em lojas de shoppings (FIALKOWSKI, 2017).

Na observação, a partir das referências bibliográficas, considerou-se os seguintes aspectos: a) o modo de uso da IoT, ou seja, como o produto se conecta a outros e qual a funcionalidade do produto promovida pela IoT; b) a relação entre os diferentes dispositivos, ou uso combinado, caracterizando a quais outros dispositivos o produto se conecta; e c) os aspectos relevantes de Design, sendo a comunicação existente sobre as funcionalidades dentro do conceito de IoT e as soluções de interação das funcionalidades da IoT do produto com os usuários.

\section{ESTUDO DE CAMPO: PRODUTOS DE LINHA BRANCA NO BRASIL QUE USAM DO CONCEITO DE IOT}

Com base na pesquisa de campo, foram encontrados 11 produtos do mercado nacional categorizados como pertencentes à linha branca, que incorporam alguma tecnologia baseada em IoT, como forma de diferenciação mercadológica, agregando valor para o produto perante o consumidor.

Considerando-se os parâmetros estabelecidos para a análise, a Tabela 1, a seguir, demonstra o resultado das informações levantadas a respeito de cada produto encontrado.

Tabela 1. Resultado do estudo de campo sob os parâmetros: modo de uso da IoT, relação entre os diferentes dispositivos e aspectos relevantes de Design. Fonte: Autoras, com base em Fialkowski (2017), Electrolux (2017 a 2017 b, 2017 c), Brastemp (2017 a, 2017 b), Midea (2017), LG (2017) e Samsung (2017).

\begin{tabular}{|c|c|c|c|c|}
\hline 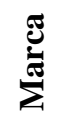 & Produto/modelo & Modo de uso & $\begin{array}{c}\text { Relação } \\
\text { entre } \\
\text { aparelhos }\end{array}$ & Design \\
\hline
\end{tabular}




\begin{tabular}{|c|c|c|c|c|}
\hline \multirow{3}{*}{ 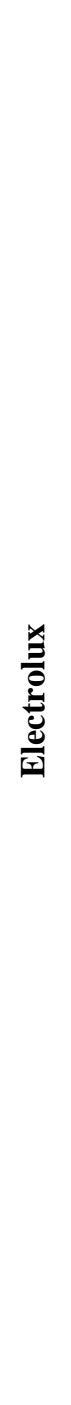 } & $\begin{array}{c}\text { Refrigerador } \\
\text { frost free modelo } \\
\text { DT52X }\end{array}$ & $\begin{array}{l}\text { QR code para lista de } \\
\text { compras. }\end{array}$ & $\begin{array}{l}\text { Entre } \\
\text { refrigerador e } \\
\text { smartphones } \\
\text { ou tablets. }\end{array}$ & $\begin{array}{l}\text { Explora a comunicação do benefício } \\
\text { para o consumidor no material de venda } \\
\text { (lojas físicas) e internet. Explora e } \\
\text { comunica a função apenas através da } \\
\text { tela touch screen. O produto está } \\
\text { normalmente desligado nos pontos de } \\
\text { venda, o que também não permite a } \\
\text { comunicação do próprio produto a esse } \\
\text { respeito nas lojas físicas. }\end{array}$ \\
\hline & $\begin{array}{l}\text { Lava \& Seca } \\
\text { modelos LSW12 } \\
\\
\text { Lava \& Seca } \\
\text { modelos LSW15 }\end{array}$ & $\begin{array}{c}\text { Controle do } \\
\text { funcionamento do } \\
\text { produto via smartphone } \\
\text { ou tablet (iOS e } \\
\text { Android), criando e } \\
\text { compartilhando } \\
\text { programas preferidos. } \\
\text { Também é possível } \\
\text { acompanhar o processo } \\
\text { de lavagem e secagem à } \\
\text { distância. }\end{array}$ & $\begin{array}{l}\text { Entre lava e } \\
\quad \text { seca e } \\
\text { smartphones } \\
\text { ou tablets. }\end{array}$ & $\begin{array}{l}\text { Observou-se a comunicação do } \\
\text { benefício para o consumidor no material } \\
\text { de venda (lojas físicas) e internet. A } \\
\text { comunicação no produto não é bem } \\
\text { explorada porque tem apenas um ícone } \\
\text { no painel indicando a possibilidade } \\
\text { desta função. Na parte posterior do } \\
\text { produto permite conectar o “acessório } \\
\text { com conectividade", que após sua } \\
\text { compra e instalação permite o uso via } \\
\text { Wi-Fi. A peça após instalada também } \\
\text { não fica visível, já que fica na parte } \\
\text { posterior do produto. }\end{array}$ \\
\hline & $\begin{array}{l}\text { Forno de micro- } \\
\text { ondas modelo } \\
\text { MT30S }\end{array}$ & $\begin{array}{l}\text { QR code para acessar } \\
\text { informações e receitas } \\
\text { recomendadas }\end{array}$ & $\begin{array}{l}\text { Entre micro- } \\
\text { ondas e } \\
\text { smartphones } \\
\text { ou tablets. }\end{array}$ & $\begin{array}{c}\text { Não se observou o benefício sendo } \\
\text { explorado nos materiais de venda e lojas } \\
\text { físicas, o benefício é apenas mencionado } \\
\text { no site da empresa. No produto o } \\
\text { benefício também é pouco comunicado } \\
\text { porque o QR code fica na parte interna } \\
\text { da porta do produto, sendo visto e } \\
\text { acessado somente ao abrir a porta. } \\
\text { Também não possui comunicação a } \\
\text { respeito no painel de comando do } \\
\text { produto. }\end{array}$ \\
\hline \multirow{2}{*}{ 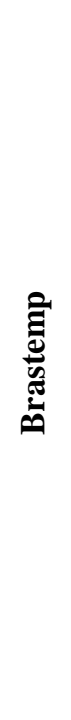 } & $\begin{array}{c}\text { Geladeira } \\
\text { modelo } \\
\text { BRN80AK Side } \\
\text { Inverse com Wi- } \\
\text { Fi }\end{array}$ & $\begin{array}{l}\text { Conexão via Wi-Fi, esta } \\
\text { já está embutida no } \\
\text { produto e permite } \\
\text { controlar e programar o } \\
\text { produto a distância. }\end{array}$ & $\begin{array}{c}\text { Entre } \\
\text { refrigerador e } \\
\text { smartphones } \\
\text { ou tablets. }\end{array}$ & $\begin{array}{l}\text { Explora a comunicação do benefício } \\
\text { para o consumidor no material de venda } \\
\text { (lojas físicas) e internet. Explora e } \\
\text { comunica bem o benefício no produto } \\
\text { com sua tela touch screen. Entretanto, } \\
\text { essa comunicação na tela não é } \\
\text { percebida no ponto de venda porque o } \\
\text { produto normalmente fica desligado. }\end{array}$ \\
\hline & $\begin{array}{l}\text { Fogão modelo } \\
\text { BFD5SAR } 5 \\
\text { Bocas Smart } \\
\text { Cook Piso }\end{array}$ & $\begin{array}{c}\text { Controle do } \\
\text { funcionamento do } \\
\text { produto via smartphone } \\
\text { ou tablet (iOS e } \\
\text { Android). Instalação da } \\
\text { conectividade é } \\
\text { posterior à compra do } \\
\text { produto. }\end{array}$ & $\begin{array}{c}\text { Entre } \\
\text { refrigerador e } \\
\text { smartphones } \\
\text { ou tablets. }\end{array}$ & $\begin{array}{l}\text { Explora a comunicação do benefício } \\
\text { para o consumidor no material de venda } \\
\text { (lojas físicas) e internet. Explora e } \\
\text { comunica a função apenas através da } \\
\text { tela touch screen. O produto está } \\
\text { normalmente desligado nos pontos de } \\
\text { venda, o que também não permite a } \\
\text { comunicação do próprio produto a esse } \\
\text { respeito nas lojas físicas. }\end{array}$ \\
\hline
\end{tabular}




\begin{tabular}{|c|c|c|c|c|}
\hline$\stackrel{g}{\stackrel{g}{g}}$ & $\begin{array}{l}\text { Ar-condicionado } \\
\text { modelo Liva } \\
\text { Inverter Wi-Fi }\end{array}$ & $\begin{array}{l}\text { Controle do } \\
\text { funcionamento do } \\
\text { produto via smartphone } \\
\text { ou tablet (iOS e } \\
\text { Android). Possível } \\
\text { comandar e acompanhar } \\
\text { a climatização do } \\
\text { ambiente à distância. }\end{array}$ & $\begin{array}{l}\text { Entre ar- } \\
\text { condicionado } \\
\mathrm{e} \\
\text { smartphones } \\
\text { ou tablets. }\end{array}$ & $\begin{array}{l}\text { Explora a comunicação do benefício } \\
\text { para o consumidor no material de venda } \\
\text { (lojas físicas) e internet. Não explora a } \\
\text { comunicação no produto e nem no } \\
\text { controle remoto do mesmo, não possui } \\
\text { ícone no produto indicando o nome } \\
\text { desta função. Na parte interior do } \\
\text { produto permite conectar o "Kit Wi-Fi } \\
\text { ready" da empresa, que após sua compra } \\
\text { e instalação permite o uso via Wi-Fi. } \\
\text { Esta peça, após instalada, também fica } \\
\text { escondida na parte interior do produto e } \\
\text { é comunicada apenas com grafismos na } \\
\text { própria peça. }\end{array}$ \\
\hline صِ & $\begin{array}{c}\text { Lava \& Seca } \\
\text { modelo Top Gun } \\
\text { 14Kg Branca LG } \\
6 \text { Motion } \\
\\
\text { Lava e Seca } \\
\text { modelo Mega } \\
\text { Touch } \\
\text { WD9WE6 }\end{array}$ & $\begin{array}{l}\text { Usa a tecnologia "NFC } \\
\text { Tag on" por meio da } \\
\text { qual pode ser feito } \\
\text { download de novos } \\
\text { programas de lavagem } \\
\text { do produto via } \\
\text { smartphone ou tablet } \\
\text { (iOS e Android). Com a } \\
\text { tecnologia "Smart } \\
\text { diagnosis" é possível } \\
\text { receber um diagnóstico } \\
\text { de possíveis problemas. }\end{array}$ & $\begin{array}{c}\text { Entre } \\
\text { lavadoras } \\
\text { com } \\
\text { smartphones } \\
\text { que possuam } \\
\text { opção para } \\
\text { essas } \\
\text { tecnologias. }\end{array}$ & $\begin{array}{c}\text { Observou-se que a comunicação do } \\
\text { benefício para o consumidor no material } \\
\text { de venda (lojas físicas) e internet é } \\
\text { pequena. Explora pouco também as } \\
\text { referidas tecnologias no produto, já que } \\
\text { não possui uma tecla ou espaço } \\
\text { exclusivo para acionar as tecnologias. } \\
\text { Esse acionamento acontece por uma } \\
\text { combinação de teclas de outras funções } \\
\text { e tempos, somente lendo o manual do } \\
\text { produto para compreender o } \\
\text { acionamento dos mesmos pelo } \\
\text { consumidor. }\end{array}$ \\
\hline 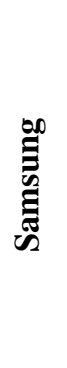 & $\begin{array}{c}\text { Lava \& Seca } \\
\text { modelo } \\
\text { WD7000 } \\
\\
\text { Lava \& Seca } \\
\text { modelo } \\
\text { WD6000 }\end{array}$ & $\begin{array}{c}\text { Com a tecnologia } \\
\text { "Smart Check" é } \\
\text { possível receber um } \\
\text { diagnóstico de possíveis } \\
\text { problemas no produto. }\end{array}$ & $\begin{array}{l}\text { Entre lava e } \\
\text { seca e } \\
\text { smartphones } \\
\text { que possam } \\
\text { instalar o } \\
\text { aplicativo da } \\
\text { empresa. }\end{array}$ & $\begin{array}{l}\text { Observou-se que a comunicação do } \\
\text { benefício para o consumidor no material } \\
\text { de venda (lojas físicas) e internet é } \\
\text { pequena. Explora pouco também a } \\
\text { referida tecnologia no produto, já que } \\
\text { não possui uma tecla ou espaço especial } \\
\text { para acionar a mesma, somente um } \\
\text { código de erro aparece no visor do } \\
\text { produto. }\end{array}$ \\
\hline
\end{tabular}

Em primeiro lugar, com relação aos dados constantes da Tabela 1, acima, a incidência do uso de IoT dentre os produtos das marcas pesquisas apresentam: Electrolux, com 4 produtos diferentes; Brastemp 2; Midea; 1, LG, 2; e Samsung, 2 produtos.

Em relação à categoria de produtos encontrados, 6 produtos são do tipo lava e seca, 2 são refrigeradores, 1 é ar-condicionado, 1 é micro-ondas e 1 é fogão. Porém, ainda assim, alguns modelos oferecem a tecnologia de IoT como um opcional a ser adquirido posteriormente à compra do produto, como é o caso do ar-condicionado da empresa Midea e da lava e seca da empresa Electrolux.

Quanto ao uso do IoT nos produtos encontrados, observou-se que a predominância das relações entre smartphones e outros aparelhos de comunicação com os produtos de linha branca, é feita, principalmente, por meio da tecnologia Bluetooth e, em menor incidência, com o uso de códigos QR. Percebe-se também que a relação entre comunicação dos usuários/produtos, apresentada pelo design até então desenvolvido e aplicada nesses dispositivos, encontra-se pouco explorada. 


\section{DISCUSSÃO}

Por meio do estudo de campo, foi possível verificar que o volume dos produtos com aplicação das tecnologias de IoT no mercado nacional de linha branca atual, encontra-se baixo em relação ao universo total de produtos existentes, como era esperado, pelos estudos teóricos analisados. Isso demonstra, portanto, pouca incidência no uso da IoT, evidenciando assim que o campo para sua aplicação em produtos de linha branca ainda está pouco explorado.

Foi possível notar também uma concentração em produtos de maior valor agregado, como lavadoras e refrigeradores que, por terem essa característica, podem embutir o uso desta tecnologia emergente no seu valor final. Esse dado não foi encontrado na revisão da literatura, indicando uma lacuna a ser pesquisada.

Sob o aspecto do modo de uso, primeiramente, pode ser visto que os cruzamentos das tecnologias integram os objetos entre os ambientes físico e virtual, visto que todos os exemplos observados no mercado nacional usam da internet para acessar ou monitorar os produtos físicos, bem como apresentam interfaces para comunicação com o usuário, confirmando os dados da literatura.

Esse cruzamento das tecnologias mostrou que se destacam dentre as formas de incorporação da IoT aplicativos relacionados às funções de controle do funcionamento do produto, sua programação e certo acompanhamento a distância. Pode-se afirmar, com base na literatura que existe ainda um campo largo a ser explorado.

Notou-se também que as informações sobre o modo de uso do consumidor não voltam à empresa que desenvolveu o mesmo, diferentemente do que aponta a literatura. Portanto, perde-se uma oportunidade de identificar padrões e comportamentos que poderiam auxiliar em melhorias para o desenvolvimento de novos produtos.

Ademais, nesses produtos, percebe-se que a unidade básica de hardware apresenta ao menos uma unidade de processamento, uma unidade de memória (smartphone ou tablets) e uma unidade de comunicação (aplicativo ou site). A transmissão das informações coletadas ocorre por meio do compartilhamento por Wi-Fi, Bluetooth e $Q R$ Codes, confirmando dados coletados na pesquisa bibliográfica.

Com respeito à relação entre os diferentes dispositivos, observa-se apenas a comunicação entre smartphones ou tablets com os eletrodomésticos. Dessa forma, apresenta-se uma limitação, como atestam os autores, uma vez que essa relação poderia ser ampliada entre os aparelhos de forma a maximizar os resultados para o consumidor, mas este potencial não é apresentado.

Por fim, quanto ao design, entende-se que o desenvolvimento de produtos e/ou serviços que propõem o uso deste conceito de conectividade, deve estar, primeiramente, pautado no usuário e prover uma experiência positiva. Porém, é notável que os produtos analisados abordam de forma superficial os benefícios que a IoT pode oferecer, pois possuem diversos problemas relativos à interação do usuário com o funcionamento dessa tecnologia. Como exemplo, tem-se alguns produtos que não apresentam IoT incorporado, fazendo com que os consumidores comprem e instalem esta posteriormente à sua aquisição. Outro exemplo é que a comunicação do benefício da IoT não fica explicita no produto, sendo necessário a consulta ao seu manual.

\section{CONSIDERAÇÕES FINAIS}


A partir dos resultados obtidos neste estudo identificou-se como tendência a incidência do uso da IoT em produtos de maior valor agregado. Quanto ao modo de uso, se destacaram os aplicativos relacionados às funções de controle, programação e tempo. E no que se refere a relação entre aparelhos, verifica-se a predominância das relações entre smartphones e outros aparelhos de comunicação com os produtos de linha branca, em que a tecnologia de Bluetooth é evidente.

O resultado do estudo mostrou que as empresas de linha branca fazem uso de seus meios de comunicação explorando a incorporação desta tecnologia, sem, no entanto, realmente isso ficar evidente no design do produto.

Desta forma, evidencia-se que esses pontos acarretam em uma percepção e experiência pouco explorada pelo usuário perante os produtos de linha branca no mercado nacional.

Cabe ressaltar que os temas abordados neste estudo estão longe de seus esgotamentos. Estes requerem ser estudados em maior profundidade, uma vez verificada oportunidade mercadológica que o uso da IoT em linha branca apresenta.

\section{REFERÊNCIAS}

BATTAIOLA, A. L. et al. Tecnologias persuasivas: A persuasão em eletrodomésticos conectados à internet. Congresso Brasileiro de Pesquisa e Desenvolvimento em Design, 2014.

BRASTEMP. Disponível em: <https://www.brastemp.com.br/produto/geladeira-brastempside-inverse-platinum-c-central-inteligente-5401/\#tenho=nao>. Acessado em 26 fev 2017 a.

BRASTEMP. Disponível em: <https://www.brastemp.com.br/produto/fogao-brastemp-ativesmart-cook-piso-5-bocas/>. Acessado em 26 fev 2017 b.

ELECTROLUX. Disponível

em:

<www.electrolux.com.br/Produtos/Refrigera\%C3\%A7\%C3\%A3o/Geladeiras---

Refrigeradores/DT52X/>. Acessado em 26 fev 2017 a.

ELECTROLUX. Disponível em: <www.electrolux.com.br/Produtos/Lavanderia/>. <http://www.electrolux.com.br/Produtos/Cozinha/Micro-ondas/MT30S/>. Acessado em 26 fev 2017 b.

ELECTROLUX. Disponível em: <http://www.electrolux.com.br/Produtos/Cozinha/Microondas/MT30S/>. Acessado em 26 fev 2017 c.

CANTANHEDE, R. F.; SILVA, C.E. Uma Proposta de Sistema de IoT para Monitoramento de Ambiente Hospitalar. Anais da VII Escola de Computação e suas Aplicações - Epoca, 2014.

DESBARATS, G. Silo-Busting to create great experiences for Internet of Things. Design Management Review. Vol. 28, Issue 1, 2017.

FIALKOWSKI, V. P. Relatório de pesquisa de campo nas lojas de eletroeletrônicos Pontos Frio e Fast Shop. Curitiba, 2017. 9 f. Trabalho não publicado.

GASPARETTO, D. A. et al. Design conectado: por um mundo de experiências. Estudos em Design| Revista (online). Rio de Janeiro: v. 24 | n. 2 [2016], p. 112 -131| ISSN 1983-196X

GIL, A. C. Como Elaborar Projetos de Pesquisa. 4 ed. São Paulo: Atlas, 2002. 175p.

IBGE. Pesquisa nacional por amostra de domicílio: Acesso à Internet e à Televisão e Posse de Telefone Móvel Celular para Uso Pessoal 2014. Disponível em: 
<http://www.ibge.gov.br/home/estatistica/pesquisas/pesquisa_resultados.php?id_pesquisa=40 $>$ Acesso em maio de 2017.

LEE, E. Y. et al. Inovação no design contemporâneo: Aproximações conceituais entre a Engenharia Kansei e a Internet das coisas. Curitiba, 2017. Trabalho não publicado.

LG. Disponível em http:// www.lg.com/br/eletrodomesticos.com/br. Acessado em 26 fev 2017.

MIDEA. Disponível em http://www.mideadobrasil.com.br/pt/produtos/interna/328/midealiva-inverter-wi-fi-frio-12.000-btu-h. Acessado em 26 fev 2017.

SAMSUNG. Disponível em http://www.samsung.com/br/home-appliances/. Acessado em 26 fev 2017.

SANTOS, B. P. et al. Internet das coisas: da teoria à prática. Departamento de Ciência da Computação - Instituto de Ciências Exatas Universidade Federal de Minas Gerais (UFMG) Belo Horizonte, MG $<$ http://homepages.dcc.ufmg.br/ mmvieira/cc/papers/internet-das-coisas.pdf > Acesso em maio de 2017. 